\title{
New existence theorems of coincidence points approach to generalizations of Mizoguchi-Takahashi's fixed point theorem
}

\author{
Ing-Jer Lin* and Tai-Hung Chen
}

\section{"Correspondence:}

ijlin@nknucc.nknu.edu.tw Department of Mathematics, National Kaohsiung Normal University, Kaohsiung, 824, Taiwan

\begin{abstract}
In this paper, we first establish some new existence theorems of coincidence points and common fixed points for $\mathcal{M T}$-functions. By applying our results, we obtain some generalizations of Mizoguchi-Takahashi's fixed point theorem, Nadler's fixed point theorem and the Banach contraction principle. Some examples illustrating our results are also given. Our results generalize and improve some main results in the literature and references therein.

Keywords: coincidence point; common fixed point; $\tau$-function; $\tau^{0}$-function; $\tau^{0}$-metric; $\mathcal{M T}$-function; Mizoguchi-Takahashi's fixed point theorem; Nadler's fixed point theorem; Banach contraction principle
\end{abstract}

\section{Introduction}

In recent years, the celebrated Banach contraction principle (see, e.g., [1]) always plays an essential role in various fields of applied mathematical analysis. The Banach contraction principle has been employed to solve the problems in Banach spaces such as the existence of solutions for nonlinear integral equations and nonlinear differential equations. Also, it has been applied to study the convergence of algorithms in computational mathematics. Additionally, many generalizations of the Banach contraction principle in various different directions have been investigated by several authors in the past; see [1-22]. Because of the importance of the Banach contraction principle, we begin with the theorem as follows.

Theorem BCP (Banach [1]) Let $(X, d)$ be a complete metric space and $T: X \rightarrow X$ be a selfmap. Assume that there exists a nonnegative number $\gamma<1$ such that

$$
d(T x, T y) \leq \gamma d(x, y) \quad \text { for all } x, y \in X
$$

Then $T$ has a unique fixed point in $X$. Moreover, for each $x \in X$, the iterative sequence $\left\{T^{n} x\right\}_{n \in \mathbb{N}}$ converges to the fixed point.

In 1969, Nadler [2] first gave a famous generalization of the Banach contraction principle for multivalued maps, which is as important as the Banach contraction principle.

\section{Springer}

(c) 2012 Lin and Chen; licensee Springer. This is an Open Access article distributed under the terms of the Creative Commons Attribution License (http://creativecommons.org/licenses/by/2.0), which permits unrestricted use, distribution, and reproduction in any medium, provided the original work is properly cited. 
Theorem NA (Nadler [2]) Let $(X, d)$ be a complete metric space and $T: X \rightarrow C B(X)$ be a $k$-contraction; that is, there exists a nonnegative number $k<1$ such that

$$
H(T x, T y) \leq k d(x, y) \quad \text { for all } x, y \in X,
$$

where $C B(X)$ is the class of all nonempty closed bounded subsets of $X$. Then there exists $v \in X$ such that $v \in T v$.

In 1989, Mizoguchi and Takahashi [3] proved a generalization of Nadler's fixed point theorem which also gave a partial answer to Problem 9 in Reich [4-6]. It is worth mentioning that the primitive proof of Mizoguchi-Takahashi's fixed point theorem is difficult. Recently, Suzuki [7] gave a very simple proof of Mizoguchi-Takahashi's fixed point theorem.

Theorem MT (Mizoguchi and Takahashi [3]) Let $(X, d)$ be a complete metric space and $T: X \rightarrow C B(X)$ be a multivalued map. Assume that

$$
H(T x, T y) \leq \alpha(d(x, y)) d(x, y) \quad \text { for all } x, y \in X,
$$

where $\alpha$ is a function from $[0, \infty)$ into $[0,1)$ satisfying $\lim \sup _{s \rightarrow t^{+}} \alpha(s)<1$ for all $t \in[0, \infty)$. Then there exists $v \in X$ such that $v \in T v$.

Subsequently, in 2007, Berinde and Berinde [8] proved the following interesting fixed point theorem. That is a generalization of Mizoguchi-Takahashi's fixed point theorem.

Theorem BB (Berinde and Berinde [8]) Let $(X, d)$ be a complete metric space, $T: X \rightarrow$ $C B(X)$ be a multivalued map, and $L \geq 0$. Assume that

$$
H(T x, T y) \leq \alpha(d(x, y)) d(x, y)+L d(y, T x) \quad \text { for all } x, y \in X,
$$

where $\alpha$ is a function from $[0, \infty)$ into $[0,1)$ satisfying $\lim \sup _{s \rightarrow t+} \alpha(s)<1$ for all $t \in[0, \infty)$. Then there exists $v \in X$ such that $v \in T$.

It is obvious that if we take $L=0$ in Berinde and Berinde's fixed point theorem, we can obtain Mizoguchi-Takahashi's fixed point theorem.

Very recently, $\mathrm{Du}$ [9] has used a $\tau^{0}$-metric and an $\mathcal{M T}$-function to establish some new fixed point theorems for nonlinear multivalued contractive maps and generalize the Banach contraction principle, Nadler's fixed point theorem, Mizoguchi-Takahashi's fixed point theorem, Berinde-Berinde's fixed point theorem, Kannan's fixed point theorems and Chatterjea's fixed point theorems for nonlinear multivalued contractive maps in complete metric spaces; see [9] for more detail.

In this paper, we first establish some new existence results of coincidence points and common fixed points for $\mathcal{M T}$-functions. By applying our results, we can obtain some generalizations of Mizoguchi-Takahashi's fixed point theorem, Nadler's fixed point theorem and the Banach contraction principle. Our results generalize and improve some main results in the literature and references therein. 


\section{Preliminaries}

Throughout this paper, we denote the set of positive integers by $\mathbb{N}$. Let $(X, d)$ be a metric space. For each $x \in X$ and $A \subseteq X$, let $d(x, A)=\inf _{y \in A} d(x, y)$. Also, we denote the class of all nonempty subsets of $X$ by $N(X)$, the family of all nonempty closed subsets of $X$ by $C(X)$, and the family of all nonempty closed and bounded subsets of $X$ by $C B(X)$. A function $H: C B(X) \times C B(X) \rightarrow[0, \infty)$ defined by

$$
H(A, B)=\max \left\{\sup _{x \in B} d(x, A), \sup _{x \in A} d(x, B)\right\}
$$

is said to be the Hausdorff metric on $C B(X)$ induced by the metric $d$ on $X$.

Let $f: X \rightarrow X$ be a selfmap and $T: X \rightarrow N(X)$ be a multivalued map. A point $v \in X$ is called

(i) a fixed point of $f$ if $f(v)=v$;

(ii) a fixed point of $T$ if $v \in T(v)$;

(iii) a coincidence point of $f$ and $T$ in $X$ if $f(v) \in T(v)$;

(iv) a common fixed point of $f$ and $T$ if $v=f(v) \in T(v)$.

In [9], Sajath and Vijayaraju proved the following theorem.

Theorem 2.1 [10] Let $(X, d)$ be a metric space, and $\alpha:(0, \infty) \rightarrow[0,1)$ be a function such that $\limsup _{r \rightarrow t^{+}} \alpha(r)<1$ for every $t \in[0, \infty)$. If $f: X \rightarrow X$ and $T: X \rightarrow C B(X)$ satisfy

(a) $H(T(x), T(y)) \leq \alpha(d(f x, f y)) d(f x, f y) \forall x, y \in X$;

(b) $T(X)=\bigcup_{x \in X} T(x) \subseteq f(X)$;

(c) $f(X)$ is a complete subspace of $X$,

then $T$ and $f$ have a coincidence point in $X$.

Remark 2.1 In fact, the condition (a) in Theorem 2.1 should be corrected as

(a) $H(T(x), T(y)) \leq \alpha(d(f x, f y)) d(f x, f y) \forall x, y \in X$ with $x \neq y$.

Moreover, it is worth mentioning that the proof of Theorem 2.1 is not correct.

The following is the definition of a $\tau$-function which was introduced and studied by Lin and Du.

Definition 2.1 $[9,11-17]$ Let $(X, d)$ be a metric space. A function $p: X \times X \rightarrow[0, \infty)$ is said to be a $\tau$-function if the following conditions hold:

( $\tau 1) ~ p(x, z) \leq p(x, y)+p(y, z)$ for all $x, y, z \in X$;

( $\tau 2)$ If $x \in X$ and $\left\{y_{n}\right\}$ in $X$ with $\lim _{n \rightarrow \infty} y_{n}=y$ such that $p\left(x, y_{n}\right) \leq M$ for some $M=M(x)>$ 0 , then $p(x, y) \leq M$

( $\tau 3)$ For any sequence $\left\{x_{n}\right\}$ in $X$ with $\lim _{n \rightarrow \infty} \sup \left\{p\left(x_{n}, x_{m}\right): m>n\right\}=0$, if there exists a sequence $\left\{y_{n}\right\}$ in $X$ such that $\lim _{n \rightarrow \infty} p\left(x_{n}, y_{n}\right)=0$, then $\lim _{n \rightarrow \infty} d\left(x_{n}, y_{n}\right)=0$;

( $\tau 4)$ For $x, y, z \in X, p(x, y)=0$ and $p(x, z)=0$ imply $y=z$.

Let $p: X \times X \rightarrow[0, \infty)$ be a $\tau$-function. Define $p(x, A)=\inf _{y \in A} p(x, y)$.

The following results are crucial and useful in this paper.

Lemma $2.1[9,11,12,14-17]$ Let $(X, d)$ be a metric space and $p: X \times X \rightarrow[0, \infty)$ be any function satisfying ( $\tau 3)$. If $\left\{x_{n}\right\}$ is a sequence in $X$ with $\lim _{n \rightarrow \infty} \sup \left\{p\left(x_{n}, x_{m}\right): m>n\right\}=0$, then $\left\{x_{n}\right\}$ is a Cauchy sequence in $X$. 
Recently, Du [5, 6] first introduced the concepts of $\tau^{0}$-functions and $\tau^{0}$-metrics as follows.

Definition 2.2 $[9,13]$ Let $(X, d)$ be a metric space. A function $p: X \times X \rightarrow[0, \infty)$ is called a $\tau^{0}$-function if it is a $\tau$-function on $X$ with $p(x, x)=0$ for all $x \in X$.

Remark 2.3 From $(\tau 4)$, if $p$ is a $\tau^{0}$-function, then $p(x, y)=0$ if and only if $x=y$.

Definition 2.3 $[9,13]$ Let $(X, d)$ be a metric space and $p$ be a $\tau^{0}$-function. For any $A, B \in$ $C B(X)$, define a function $D_{p}: C B(X) \times C B(X) \rightarrow[0, \infty)$ by

$$
D_{p}(A, B)=\max \left\{\delta_{p}(A, B), \delta_{p}(B, A)\right\},
$$

where $\delta_{p}(A, B)=\sup _{x \in A} p(x, B)$; then $D_{p}$ is said to be a $\tau^{0}$-metric on $\mathrm{CB}(\mathrm{X})$ induced by $p$.

Clearly, any Hausdorff metric is a $\tau^{0}$-metric, but the reverse is not true.

Definition 2.4 $[9,15-22]$ A function $\varphi:[0, \infty) \rightarrow[0,1)$ is said to be an $\mathcal{M T}$-function (or an $\mathcal{R}$-function) if $\limsup _{s \rightarrow t^{+}} \varphi(s)<1$ for all $t \in[0, \infty)$.

Lemma 2.2 [9] Let $\varphi:[0, \infty) \rightarrow[0,1)$ be an $\mathcal{M T}$-function. Then $\kappa:[0, \infty) \rightarrow[0,1)$ defined by $\kappa(t)=\frac{\varphi(t)+1}{2}$ is also an $\mathcal{M T}$-function.

Theorem D [22] Let $\varphi:[0, \infty) \rightarrow[0,1)$ be a function. Then the following statements are equivalent.

(a) $\varphi$ is an $\mathcal{M T}$-function.

(b) For each $t \in[0, \infty)$, there exist $r_{t}{ }^{(1)} \in[0,1)$ and $\epsilon_{t}{ }^{(1)}>0$ such that $\varphi(s) \leq r_{t}{ }^{(1)}$ for all $s \in\left(t, t+\epsilon_{t}^{(1)}\right)$.

(c) For each $t \in[0, \infty)$, there exist $r_{t}^{(2)} \in[0,1)$ and $\epsilon_{t}{ }^{(2)}>0$ such that $\varphi(s) \leq r_{t}{ }^{(2)}$ for all $s \in\left[t, t+\epsilon_{t}^{(2)}\right]$.

(d) For each $t \in[0, \infty)$, there exist $r_{t}^{(3)} \in[0,1)$ and $\epsilon_{t}{ }^{(3)}>0$ such that $\varphi(s) \leq r_{t}{ }^{(3)}$ for all $s \in\left(t, t+\epsilon_{t}^{(3)}\right]$.

(e) For each $t \in[0, \infty)$, there exist $r_{t}{ }^{(4)} \in[0,1)$ and $\epsilon_{t}{ }^{(4)}>0$ such that $\varphi(s) \leq r_{t}{ }^{(4)}$ for all $s \in\left[t, t+\epsilon_{t}^{(4)}\right)$.

(f) For any nonincreasing sequence $\left\{x_{n}\right\}_{n \in \mathbb{N}}$ in $[0, \infty)$, we have $0 \leq \sup _{n \in \mathbb{N}} \varphi\left(x_{n}\right)<1$.

(g) $\varphi$ is a function of a contractive factor [19]; that is, for any strictly decreasing sequence $\left\{x_{n}\right\}_{n \in \mathbb{N}}$ in $[0, \infty)$, we have $0 \leq \sup _{n \in \mathbb{N}} \varphi\left(x_{n}\right)<1$.

It is obvious that if a function $\alpha:[0, \infty) \rightarrow[0,1)$ is nondecreasing or nonincreasing, then it is an $\mathcal{M T}$-function.

\section{New coincidence point theorems and a common fixed point theorem}

In this section, we generalize Theorem 2.1 which is one of the main results in [10]. Please notice that our proof is quite different from the proof of Theorem 2.1 in [10].

Theorem 3.1 Let $(X, d)$ be a metric space, $p: X \times X \rightarrow[0, \infty)$ be a $\tau^{0}$-function, $D_{p}$ be a $\tau^{0}$-metric on $C B(X)$ induced by $p$ and $\varphi:[0, \infty) \rightarrow[0,1)$ be an $\mathcal{M T}$-function. If $T: X \rightarrow$ $C B(X)$ and $f: X \rightarrow X$ satisfy 
(i) $D_{p}(T(x), T(y)) \leq \varphi(p(f(x), f(y))) p(f(x), f(y)), \forall x, y \in X$;

(ii) $T(X)=\bigcup_{x \in X} T(x) \subseteq f(X)$;

(iii) $f(X)$ is a complete subspace of $X$,

then $T$ and $f$ have a coincidence point in $X$.

Proof By Lemma 2.2, we can define an $\mathcal{M T}$-function $\kappa:[0, \infty) \rightarrow[0,1)$ by $\kappa(t)=\frac{\varphi(t)+1}{2}$. Then $\varphi(t)<\kappa(t)$ and $0<\kappa(t)<1$ for all $t \in[0, \infty)$. Let $x_{0} \in X$. By (ii), there exists $x_{1} \in X$ such that $f\left(x_{1}\right) \in T\left(x_{0}\right)$. If $f\left(x_{0}\right)=f\left(x_{1}\right)$, we have $f\left(x_{0}\right) \in T\left(x_{0}\right)$ which means that $x_{0}$ is a coincidence point of $T$ and $f$ in $X$ and we finish the proof. Otherwise, if $f\left(x_{0}\right) \neq f\left(x_{1}\right)$, since $p$ is a $\tau^{0}$-function, $p\left(f\left(x_{0}\right), f\left(x_{1}\right)\right)>0$. By (i), we have

$$
\begin{aligned}
p\left(f\left(x_{1}\right), T\left(x_{1}\right)\right) & \leq \sup _{y \in T\left(x_{0}\right)} p\left(y, T\left(x_{1}\right)\right) \\
& \leq D_{p}\left(T\left(x_{0}\right), T\left(x_{1}\right)\right) \\
& \leq \varphi\left(p\left(f\left(x_{0}\right), f\left(x_{1}\right)\right)\right) p\left(f\left(x_{0}\right), f\left(x_{1}\right)\right) \\
& <\kappa\left(p\left(f\left(x_{0}\right), f\left(x_{1}\right)\right)\right) p\left(f\left(x_{0}\right), f\left(x_{1}\right)\right) .
\end{aligned}
$$

Hence there exists $a \in T\left(x_{1}\right)$ such that $p\left(f\left(x_{1}\right), a\right)<\kappa\left(p\left(f\left(x_{0}\right), f\left(x_{1}\right)\right)\right) p\left(f\left(x_{0}\right), f\left(x_{1}\right)\right)$. By (ii) again, there exists $x_{2} \in X$ such that $f\left(x_{2}\right)=a \in T\left(x_{1}\right)$. Therefore,

$$
p\left(f\left(x_{1}\right), f\left(x_{2}\right)\right)<\kappa\left(p\left(f\left(x_{0}\right), f\left(x_{1}\right)\right)\right) p\left(f\left(x_{0}\right), f\left(x_{1}\right)\right) .
$$

By induction, we can obtain a sequence $\left\{f\left(x_{n}\right)\right\}$ in $X$ satisfying $f\left(x_{n}\right) \in T\left(x_{n-1}\right)$ and

$$
p\left(f\left(x_{n}\right), f\left(x_{n+1}\right)\right)<\kappa\left(p\left(f\left(x_{n-1}\right), f\left(x_{n}\right)\right)\right) p\left(f\left(x_{n-1}\right), f\left(x_{n}\right)\right) \quad \forall n \in \mathbb{N} .
$$

Since $\kappa(t)<1$ for all $t \in[0, \infty)$, the inequality (3.1) implies the sequence $\left\{p\left(f\left(x_{n-1}\right)\right.\right.$, $\left.\left.f\left(x_{n}\right)\right)\right\}_{n \in \mathbb{N}}$ is strictly decreasing in $[0, \infty)$. Since $\kappa$ is an $\mathcal{M T}$-function, by Theorem $\mathrm{D}$, we have

$$
0<\sup _{n \in \mathbb{N}} \kappa\left(p\left(f\left(x_{n-1}\right), f\left(x_{n}\right)\right)\right)<1 .
$$

Let $\lambda:=\sup _{n \in \mathbb{N}} \kappa\left(p\left(f\left(x_{n-1}\right), f\left(x_{n}\right)\right)\right)$. Then $0<\lambda<1$ and $\kappa\left(p\left(f\left(x_{n-1}\right), f\left(x_{n}\right)\right)\right) \leq \lambda$ for all $n \in \mathbb{N}$. For any $n \in \mathbb{N}$, we have from (3.1) that

$$
\begin{aligned}
p\left(f\left(x_{n}\right), f\left(x_{n+1}\right)\right) & <\kappa\left(p\left(f\left(x_{n-1}\right), f\left(x_{n}\right)\right)\right) p\left(f\left(x_{n-1}\right), f\left(x_{n}\right)\right) \\
& \leq \lambda p\left(f\left(x_{n-1}\right), f\left(x_{n}\right)\right) \\
& <\lambda^{2} p\left(f\left(x_{n-2}\right), f\left(x_{n-1}\right)\right) \\
& <\cdots \\
& <\lambda^{n} p\left(f\left(x_{0}\right), f\left(x_{1}\right)\right) .
\end{aligned}
$$


Let $v_{n}=f\left(x_{n}\right)$ for all $n \in \mathbb{N} \cup\{0\}$. We claim that $\lim _{n \rightarrow \infty} \sup \left\{p\left(v_{n}, v_{m}\right): m>n\right\}=0$. Put $\alpha_{n}=\frac{\lambda^{n}}{1-\lambda} p\left(v_{0}, v_{1}\right), n \in \mathbb{N}$. For $m, n \in \mathbb{N}$ with $m>n$, by (3.2), we have

$$
p\left(v_{n}, v_{m}\right) \leq \sum_{j=n}^{m-1} p\left(v_{j}, v_{j+1}\right)<\alpha_{n} .
$$

Since $0<\lambda<1, \lim _{n \rightarrow \infty} \alpha_{n}=0$ and hence

$$
\lim _{n \rightarrow \infty} \sup \left\{p\left(v_{n}, v_{m}\right): m>n\right\}=0 .
$$

By Lemma 2.1, $\left\{v_{n}\right\}$ is a Cauchy sequence in $f(X)$. By the completeness of $f(X)$, there exists $\hat{v} \in X$ such that $v_{n} \rightarrow f(\hat{v})$ as $n \rightarrow \infty$. From $(\tau 2)$ and (3.3), we have

$$
p\left(v_{n}, f(\hat{v})\right) \leq \alpha_{n} \quad \text { for all } n \in \mathbb{N} .
$$

So, for each $n \in \mathbb{N}$, we have

$$
\begin{aligned}
p\left(v_{n+1}, T(\hat{v})\right) & =p\left(f\left(x_{n+1}\right), T(\hat{v})\right) \\
& \leq \sup _{y \in T\left(x_{n}\right)} p(y, T(\hat{v})) \\
& \leq D_{p}\left(T\left(x_{n}\right), T(\hat{v})\right) \\
& \leq \varphi\left(p\left(v_{n}, f(\hat{v})\right)\right) p\left(v_{n}, f(\hat{v})\right) \\
& <\kappa\left(p\left(v_{n}, f(\hat{v})\right)\right) p\left(v_{n}, f(\hat{v})\right) \\
& <p\left(v_{n}, f(\hat{v})\right) \\
& \leq \alpha_{n} .
\end{aligned}
$$

Therefore, there exists $y_{n+1} \in T(\hat{v})$ such that $p\left(v_{n+1}, y_{n+1}\right)<\alpha_{n}$ for each $n \in \mathbb{N}$, which implies $\lim _{n \rightarrow \infty} p\left(v_{n}, y_{n}\right)=0$. Then, by $(\tau 3)$, we have $\lim _{n \rightarrow \infty} d\left(v_{n}, y_{n}\right)=0$. Moreover, since $v_{n} \rightarrow$ $f(\hat{v})$ as $n \rightarrow \infty$ and

$$
0 \leq d\left(f(\hat{v}), y_{n+1}\right) \leq d\left(f(\hat{v}), v_{n+1}\right)+d\left(v_{n+1}, y_{n+1}\right) \quad \text { for all } n \in \mathbb{N}
$$

we get

$$
\lim _{n \rightarrow \infty} d\left(f(\hat{v}), y_{n+1}\right)=0,
$$

which means that $y_{n} \rightarrow f(\hat{v})$ as $n \rightarrow \infty$. Since $y_{n+1} \in T(\hat{v})$ for all $n \in \mathbb{N}$ and $T(\hat{v})$ is closed, $f(\hat{v}) \in T(\hat{v})$, i.e., $\hat{v}$ is a coincidence point of $f$ and $T$. The proof is completed.

Remark 3.1 In Theorem 3.1, if $f=i d$ (the identity map), then we obtain MizoguchiTakahashi's fixed point theorem. So Theorem 3.1 is a generalization of MizoguchiTakahashi's fixed point theorem, Nadler's fixed point theorem and the Banach contraction principle.

Here, we give a simple example illustrating Theorem 3.1. 
Example 3.1 Let $X=[0, \infty)$ with the metric $d(x, y)=|x-y|, x, y \in X$. Let $f(x)=2 x, T(x)=$ $[0, x]$ and $\varphi(x)=\frac{2}{3}, \forall x \in X$. Let $p: X \times X \rightarrow[0, \infty)$ be defined by

$$
p(x, y)=\max \{a(x-y), b(y-x)\}
$$

for all $x, y \in X$ and $0<a<b$. It is easy to see that $p$ is a $\tau^{0}$-function and $\varphi$ is an $\mathcal{M T}$ function.

Clearly, $T(X)=\bigcup_{x \in X} T(x) \subseteq f(X)$ and $f(X)$ is a complete subspace of $X$. We claim that $D_{p}(T(x), T(y)) \leq \varphi(p(f x, f y)) p(f x, f y), \forall x, y \in X$. Indeed, we consider the following two possible cases:

Case 1. If $0 \leq x<y$, we have $T x=[0, x]$ and $T y=[0, y]$, then

$$
p(f x, f y)=\max \{a(2 x-2 y), b(2 y-2 x)\}=2 b(y-x)
$$

and

$$
\begin{aligned}
D_{p}(T x, T y) & =\max \left\{\sup _{z \in T x} p(z, T y), \sup _{z \in T y} p(z, T x)\right\} \\
& =a(y-x) \\
& <b(y-x) \\
& <\frac{2}{3} p(f x, f y) \\
& =\varphi(p(f x, f y)) p(f x, f y) .
\end{aligned}
$$

Case 2. If $0 \leq y<x$, similarly, we have

$$
p(f x, f y)=\max \{a(2 x-2 y), b(2 y-2 x)\}=2 a(x-y) ;
$$

and

$$
\begin{aligned}
D_{p}(T x, T y) & =\max \left\{\sup _{z \in T x} p(z, T y), \sup _{z \in T y} p(z, T x)\right\} \\
& =a(x-y) \\
& <\frac{2}{3} p(f x, f y) \\
& =\varphi(p(f x, f y)) p(f x, f y) .
\end{aligned}
$$

By Cases 1 and 2, we verify that $D_{p}(T(x), T(y)) \leq \varphi(p(f x, f y)) p(f x, f y), \forall x, y \in X$. Therefore, all the assumptions of Theorem 3.1 are satisfied. So, we can apply Theorem 3.1 to show that $f$ and $T$ have a coincidence point in $X$. Actually, 0 is a coincidence point of $f$ and $T$ since $f(0) \in T(0)$.

The following result follows immediately from Theorem 3.1.

Corollary 3.1 Let $(X, d)$ be a metric space, $p: X \times X \rightarrow[0, \infty)$ be a $\tau^{0}$-function, $D_{p}$ be a $\tau^{0}$ metric on $C B(X)$ induced by $p$ and $\alpha:[0, \infty) \rightarrow[0,1)$ be a nondecreasing or nonincreasing function. If $T: X \rightarrow C B(X)$ and $f: X \rightarrow X$ satisfy 
(i) $D_{p}(T(x), T(y)) \leq \alpha(p(f(x), f(y))) p(f(x), f(y)) \forall x, y \in X$;

(ii) $T(X)=\bigcup_{x \in X} T(x) \subseteq f(X)$;

(iii) $f(X)$ is a complete subspace of $X$,

then $T$ and $f$ have a coincidence point in $X$.

In Theorem 3.1, if $p \equiv d$, then $D_{p} \equiv H$ and we have the following corollary.

Corollary 3.2 Let $(X, d)$ be a metric space and $\varphi:[0, \infty) \rightarrow[0,1)$ be an $\mathcal{M T}$-function. If $T: X \rightarrow C B(X)$ and $f: X \rightarrow X$ satisfy

(i) $H(T(x), T(y)) \leq \varphi(d(f(x), f(y))) d(f(x), f(y)) \forall x, y \in X$;

(ii) $T(X)=\bigcup_{x \in X} T(x) \subseteq f(X)$;

(iii) $f(X)$ is a complete subspace of $X$,

then $T$ and $f$ have a coincidence point in $X$.

Corollary 3.3 Let $(X, d)$ be a metric space and $\alpha:[0, \infty) \rightarrow[0,1)$ be a nondecreasing or nonincreasing function. If $T: X \rightarrow C B(X)$ and $f: X \rightarrow X$ satisfy

(i) $H(T(x), T(y)) \leq \alpha(d(f(x), f(y))) d(f(x), f(y)) \forall x, y \in X$;

(ii) $T(X)=\bigcup_{x \in X} T(x) \subseteq f(X)$;

(iii) $f(X)$ is a complete subspace of $X$,

then $T$ and $f$ have a coincidence point in $X$.

Theorem 3.2 Let $(X, d)$ be a metric space, $p: X \times X \rightarrow[0, \infty)$ be a $\tau^{0}$-function, $D_{p}$ be a $\tau^{0}$-metric on $C B(X)$ induced by $p$ and $\varphi:[0, \infty) \rightarrow[0,1)$ be an $\mathcal{M T}$-function. If $T: X \rightarrow$ $C B(X)$ and $f: X \rightarrow X$ satisfy

(i) $D_{p}(T(x), T(y)) \leq \varphi(p(f x, f y)) p(f x, f y) \forall x, y \in X$;

(ii) $T(X)=\bigcup_{x \in X} T(x) \subseteq f(X)$;

(iii) $f(X)$ is a complete subspace of $X$;

(iv) $f v=f f v$ if $v$ is a coincidence point of $f$ and $T$,

then $T$ and $f$ have a common fixed point in $X$.

Proof Following the same argument as in the proof of Theorem 3.1, we can construct two sequences $\left\{x_{n}\right\}$ and $\left\{v_{n}\right\}$ satisfying

(a) $v_{n}=f\left(x_{n}\right) \in T\left(x_{n-1}\right)$ for all $n \in \mathbb{N}$;

(b) $v_{n}$ is a Cauchy sequence in $X$ and $\lim _{n \rightarrow \infty} \sup \left\{p\left(v_{n}, v_{m}\right): m>n\right\}=0$;

(c) there exist $\hat{v} \in X$ such that

- $v_{n} \rightarrow f(\hat{v})$ as $n \rightarrow \infty$;

- $f(\hat{v}) \in T(\hat{v})$;

- $p\left(v_{n}, f(\hat{v})\right) \leq \alpha_{n}$, where $\alpha_{n}=\frac{\lambda^{n}}{1-\lambda} p\left(v_{0}, v_{1}\right), n \in \mathbb{N}$.

By (c) and (iv), we have $f \hat{v}=f f \hat{v}$. Then

$$
\begin{aligned}
p\left(v_{n}, T(f(\hat{v}))\right) & \leq \sup _{z \in T\left(x_{n-1}\right)} p(z, T(f(\hat{v}))) \\
& \leq D_{p}\left(T\left(x_{n-1}\right), T(f(\hat{v}))\right) \\
& \leq \varphi\left(p\left(f\left(x_{n-1}\right), f(f(\hat{v}))\right)\right) p\left(f\left(x_{n-1}\right), f(f(\hat{v}))\right) \\
& <p\left(f\left(x_{n-1}\right), f(f(\hat{v}))\right) .
\end{aligned}
$$


Therefore, there exists $z_{n} \in T(f(\hat{v}))$ such that

$$
p\left(v_{n}, z_{n}\right)<p\left(f\left(x_{n-1}\right), f(f(\hat{v}))\right) \quad \text { for all } n \in \mathbb{N} \text {. }
$$

Since

$$
\begin{aligned}
p\left(f\left(x_{n-1}\right), f(f(\hat{v}))\right) & \leq p\left(f\left(x_{n-1}\right), f(\hat{v})\right)+p(f(\hat{v}), f(f(\hat{v}))) \\
& \leq \alpha_{n-1},
\end{aligned}
$$

we have $\lim _{n \rightarrow \infty} p\left(f\left(x_{n-1}\right), f(f(\hat{v}))\right)=0$. By (3.6), $\lim _{n \rightarrow \infty} p\left(v_{n}, z_{n}\right)=0$. By $(\tau 3)$, we have $\lim _{n \rightarrow \infty} d\left(v_{n}, z_{n}\right)=0$. Since $v_{n} \rightarrow f(\hat{v})$ as $n \rightarrow \infty$ and

$$
d\left(f(\hat{v}), z_{n}\right) \leq d\left(f(\hat{v}), v_{n}\right)+d\left(v_{n}, z_{n}\right)
$$

we have $\lim _{n \rightarrow \infty} d\left(f(\hat{v}), z_{n}\right)=0$, which implies $\lim _{n \rightarrow \infty} z_{n}=f(\hat{v})$. Since $T(f(\hat{v}))$ is closed and $z_{n} \in T(f(\hat{v}))$ for all $n \in \mathbb{N}$, we get $f(\hat{v}) \in T(f(\hat{v}))$. Therefore, $f \hat{v}=f f \hat{v} \in T f \hat{v}$, which means that $f(\hat{v})$ is a common fixed point of $f$ and $T$ in $X$. The proof is completed.

Remark 3.2 Theorem 3.2 also generalizes and improves Mizoguchi-Takahashi's fixed point theorem.

Example 3.2 In Example 3.1, we have shown that 0 is a coincidence point of $f$ and $T$. Clearly, $0=f(0)=f(f(0))$. So, all the assumptions of Theorem 3.2 are satisfied. By Theorem 3.2, we know that $f$ and $T$ have a common fixed point in $X$. Actually, 0 is a common fixed point of $f$ and $T$ since $0=f(0) \in T(0)$.

Similarly, we have the following corollary.

Corollary 3.4 Let $(X, d)$ be a metric space, $p: X \times X \rightarrow[0, \infty)$ be a $\tau^{0}$-function, $D_{p}$ be a $\tau^{0}$ metric on $C B(X)$ induced by $p$ and $\alpha:[0, \infty) \rightarrow[0,1)$ be a nondecreasing or nonincreasing function. If $T: X \rightarrow C B(X)$ and $f: X \rightarrow X$ satisfy

(i) $D_{p}(T(x), T(y)) \leq \alpha(p(f x, f y)) p(f x, f y) \forall x, y \in X$;

(ii) $T(X)=\bigcup_{x \in X} T(x) \subseteq f(X)$;

(iii) $f(X)$ is a complete subspace of $X$;

(iv) $f v=f f v$ if $v$ is a coincidence point off and $T$,

then $T$ and $f$ have a common fixed point in $X$.

Corollary 3.5 Let $(X, d)$ be a metric space and $\varphi:[0, \infty) \rightarrow[0,1)$ be an $\mathcal{M T}$-function. If $T: X \rightarrow C B(X)$ and $f: X \rightarrow X$ satisfy

(i) $H(T(x), T(y)) \leq \varphi(d(f x, f y)) d(f x, f y) \forall x, y \in X$;

(ii) $T(X)=\bigcup_{x \in X} T(x) \subseteq f(X)$;

(iii) $f(X)$ is a complete subspace of $X$;

(iv) $f v=f f v$ if $v$ is a coincidence point off and $T$, then $T$ and $f$ have a common fixed point in $X$.

Corollary 3.6 Let $(X, d)$ be a metric space and $\alpha:[0, \infty) \rightarrow[0,1)$ be a nondecreasing or nonincreasing function. If $T: X \rightarrow C B(X)$ and $f: X \rightarrow X$ satisfy 
(i) $H(T(x), T(y)) \leq \alpha(d(f x, f y)) d(f x, f y) \forall x, y \in X$;

(ii) $T(X)=\bigcup_{x \in X} T(x) \subseteq f(X)$;

(iii) $f(X)$ is a complete subspace of $X$;

(iv) $f v=f f v$ if $v$ is a coincidence point of $f$ and $T$,

then $T$ and $f$ have a common fixed point in $X$.

\section{Competing interests}

The authors declare that they have no competing interests.

\section{Authors' contributions}

The first author made $80 \%$ contribution: problem design, coordination, discussion, revision of the important part, and submission of this paper. The second author made $20 \%$ contribution: discussion, responsibility for the important results and typing of this paper.

\section{Acknowledgements}

The authors wish to express their hearty thanks to Professor Wei-Shih Du for their valuable suggestions and comments.

Received: 24 May 2012 Accepted: 30 August 2012 Published: 19 September 2012

\section{References}

1. Takahashi, W: Nonlinear Functional Analysis. Yokohama Publishers, Yokohama (2000)

2. Nadler, SB Jr.: Multi-valued contraction mappings. Pac. J. Math. 30, 475-488 (1969)

3. Mizoguchi, N, Takahashi, W: Fixed point theorems for multivalued mappings on complete metric spaces. J. Math. Anal. Appl. 141, 177-188 (1989)

4. Reich, S: Some problems and results in fixed point theory. Contemp. Math. 21, 179-187 (1983)

5. Daffer, PZ, Kaneko, H, Li, W: On a conjecture of S. Reich. Proc. Am. Math. Soc. 124, 3159-3162 (1996)

6. Jachymski, J: On Reich's question concerning fixed points of multimaps. Boll. Unione Mat. Ital., A 9, $453-460$ (1995)

7. Suzuki, T: Mizoguchi-Takahashi's fixed point theorem is a real generalization of Nadler's. J. Math. Anal. Appl. 340, 752-755 (2008)

8. Berinde, M, Berinde, V: On a general class of multi-valued weakly Picard mappings. J. Math. Anal. Appl. 326, 772-782 (2007)

9. Du, W-S: Some new results and generalizations in metric fixed point theory. Nonlinear Anal. 73, 1439-1446 (2010)

10. Sajath, ZMI, Vijayaraju, P: Coincidence and common fixed points of hybrid mappings. Int. Math. Forum 5(42), 2063-2069 (2010)

11. Lin, L-J, Du, W-S: Ekeland's variational principle, minimax theorems and existence of nonconvex equilibria in complete metric spaces. J. Math. Anal. Appl. 323, 360-370 (2006)

12. Lin, L-J, Du, W-S: On maximal element theorems, variants of Ekeland's variational principle and their applications. Nonlinear Anal. 68, 1246-1262 (2008)

13. Du, W-S: Fixed point theorems for generalized Hausdorff metrics. Int. Math. Forum 3, 1011-1022 (2008)

14. Du, W-S: Critical point theorems for nonlinear dynamical systems and their applications. Fixed Point Theory Appl. 2010, Article ID 246382 (2010). doi:10.1155/2010/246382

15. He, Z, Du, W-S, Lin, I-J: The existence of fixed points for new nonlinear multivalued maps and their applications. Fixed Point Theory Appl. 2011, 84 (2011)

16. Du, W-S: On generalized weakly directional contractions and approximate fixed point property with applications. Fixed Point Theory Appl. 2012, 6 (2012)

17. Du, W-S, Zheng, S-X: Nonlinear conditions for coincidence point and fixed point theorems. Taiwan. J. Math. 16, 857-868 (2012)

18. Du, W-S: Coupled fixed point theorems for nonlinear contractions satisfied Mizoguchi-Takahashi's condition in quasiordered metric spaces. Fixed Point Theory Appl. 2010, Article ID 876372 (2010)

19. Du, W-S: Nonlinear contractive conditions for coupled cone fixed point theorems. Fixed Point Theory Appl. 2010, Article ID 190606 (2010)

20. Du, W-S: New cone fixed point theorems for nonlinear multivalued maps with their applications. Appl. Math. Lett. 24(2), 172-178 (2011)

21. Du, W-S: On approximate coincidence point properties and their applications to fixed point theory. J. Appl. Math. 2012, Article ID 302830 (2012). doi:10.1155/2012/302830

22. Du, W-S: On coincidence point and fixed point theorems for nonlinear multivalued maps. Topol. Appl. 159(1), 49-56 (2012)

doi:10.1186/1687-1812-2012-156

Cite this article as: Lin and Chen: New existence theorems of coincidence points approach to generalizations of

Mizoguchi-Takahashi's fixed point theorem. Fixed Point Theory and Applications 2012 2012:156. 